\title{
Architecture of a catalytically active homotrimeric plant cellulose synthase complex
}

\author{
Pallinti Purushotham*, Ruoya Ho*, Jochen Zimmer† \\ Department of Molecular Physiology and Biological Physics, University of Virginia School of Medicine, 480 Ray C. Hunt Dr., Charlottesville, VA 22903, USA. \\ *These authors contributed equally to this work. †Corresponding author. Email: jochen_zimmer@virginia.edu
}

Cellulose is an essential plant cell wall component and represents the most abundant biopolymer on Earth. Supramolecular plant cellulose synthase complexes organize multiple linear glucose polymers into microfibrils as load-bearing wall components. We determined the structure of a poplar cellulose synthase CesA homotrimer that suggests a molecular basis for cellulose microfibril formation. This complex, stabilized by cytosolic plant conserved regions and helical exchange within the transmembrane segment, forms three channels occupied by nascent cellulose polymers. Secretion steers the polymers toward a common exit point, which may facilitate protofibril formation. CesAs' $\mathrm{N}$-terminal domains assemble into a cytosolic stalk that interacts with a microtubule-tethering protein and may thus be involved in CesA localization. Our data suggest how cellulose synthase complexes assemble and provide the molecular basis for plant cell wall engineering.

A main product of plant photosynthesis is glucose, which is a precursor of many naturally occurring carbohydrates and a building block for cell wall materials in most photosynthetic organisms (1). Cellulose, produced by cellulose synthase (CesA), is a linear $\beta-1,4-l i n k e d$ glucose polymer and a fundamental cell wall component in land plants (2). With an estimated production exceeding 10 gigatons annually (3), it represents the most abundant biopolymer on earth as well as a vast carbon dioxide sink and energy resource. CesA is a membrane-embedded family-2 glycosyltransferase (GT) related to chitin, alginate and hyaluronan synthases $(4,5)$. The enzyme catalyzes glucose transfer from UDP-glucose (UDPGlc, the donor) to the terminal C4 hydroxyl of the nascent cellulose polymer (the acceptor) (fig. S1A).

CesA combines two functions: it synthesizes cellulose and secretes the polymer through a transmembrane (TM) channel formed by its membrane-spanning segment (6). The translocation mechanism has been described in detail for the bacterial homolog BcsA. Cellulose synthase uses an evolutionarily conserved 'Gating Loop' and 'Finger Helix' to couple the binding of a new substrate molecule after cellulose elongation with the translocation of the polymer into its TM channel (7). Despite similar biosynthesis mechanisms, bacterial and plant cellulose differs substantially. While most bacteria [with some exceptions (8)] produce amorphous cellulose (9), plant CesAs organize multiple cellulose chains into micro- and macrofibrils (CMFs) that are spun around the cell to form the wall's load-bearing components (10).

Most plants express several CesA isoforms with different subsets required for primary and secondary cell wall formation (10). Reconstitutions of single CesA isoforms from
Physcomitrella patens and Populus tremula $x$ tremuloides (Ptt) demonstrated that, in vitro, single isoforms devoid of any additional plant-derived factors are sufficient to form cellulose fibers resembling authentic wall CMFs $(11,12)$. CMFs are likely synthesized by supramolecular CesA complexes (CSCs) in the plasma membrane. Accordingly, plant CesAs contain three distinct regions likely mediating oligomerization. First, their N-terminal domains (NTDs) form extended cytosolic domains $~ 140-160$ residues long with a RING-like domain near the extreme $\mathrm{N}$ terminus followed by hypervariable regions leading into the first TM helix. Second, a plantconserved region (PCR, 118 residues) is inserted into the catalytic GT domain at the membrane distal side, and third, a class-specific region (CSR, $\sim 124$ residues) follows an evolutionarily conserved substrate-binding motif near the active site $(13,14)$. The isolated $\mathrm{N}$ terminus from cotton CesA1 has been shown to dimerize in vitro (15). In the plasma membrane, CesAs assemble into pseudo 6-fold symmetric CSCs, termed rosettes (16). CSCs were proposed to consist of hexamers of either trimers, tetramers or hexamers, thereby giving rise to 18,24 or 36 CesAs per CSC, respectively, synthesizing CMFs with a corresponding maximum number of cellulose chains (17).

To reveal the mechanism of plant CMF formation, we determined the cryo electron microscopy (cryo-EM) structure of a homo-oligomeric PttCesA8 complex, implicated in secondary cell wall formation in vivo (10), at an intermediate state during cellulose biosynthesis at about $3.5 \AA$ resolution. The structure reveals a trimeric PttCesA8 complex in which each enzyme is associated with a nascent polysaccharide. Our data reveal how CesA trimers are stabilized to steer cellulose 
chains into protofibrils that could align into CMFs when synthesized by a rosette consisting of six CesA trimers.

\section{Results}

\section{Homo-oligomerization of PttCesA8}

PttCesA8 was recombinantly expressed in SF9 insect cells with an N-terminal poly-histidine tag facilitating affinity purification (see Methods). The protein was solubilized from the membrane fraction in a combination of the detergents Lauryl Maltose Neopentyl Glycol (LMNG) and cholesteryl-hemisuccinate (CHS) and exchanged into glyco-diosgenin (GDN) during purification (fig. S1B). Under these conditions, the purified enzyme is catalytically active and synthesizes a water-insoluble glucan in the presence of UDP-Glc and $\mathrm{Mg}^{2+}$ or $\mathrm{Mn}^{2+}$ (fig. S1C). The polymer is completely degraded by a $\beta$ 1,4 specific glucanase, consistent with the synthesis of authentic cellulose, as also previously described for proteoliposome-reconstituted PttCesA8 (12). Although most of the in vitro synthesized cellulose is amorphous and not detectable by negative stain EM analyses, some thin fibers were observed approximately 10-15 $\AA$ in diameter, perhaps representing cellulose protofibrils containing a small number of cellulose chains (fig. S1D).

To test the ability of PttCesA8 to self-assemble into homooligomers, we co-expressed three protein variants carrying either an N-terminal tandem His-Strep-, FLAG- or Myc-tag in Pichia pastoris. Purification of the recombinant proteins by sequential binding to four affinity matrices (TALON resin, followed by anti-Strep, -FLAG and -Myc matrices) revealed the co-purification of all three PttCesA8 species in a functional form (fig. S1, E and F). Combined with our structural analyses described below, these interaction studies suggest that PttCesA8 can assemble into catalytically active homo-oligomers in vitro.

\section{Architecture of the PttCesAs homotrimer}

Structural insights into PttCesA8 homo-oligomers were obtained by cryo-EM (table S1). To minimize sample dissociation during purification, we designed a fast purification scheme consisting of immobilized metal affinity and gel filtration chromatography of the N-terminally His-tagged PttCesA8. Cryo grids were prepared immediately after purification without addition of UDP-Glc as explained in detail in Methods. The 2-dimensional classification of $\sim 3,000,000$ particles revealed the presence of small ( 85\%) and large ( 15\%) particles, in agreement with monomers and trimers, with the monomers likely arising from detergent-dissociated trimers (Fig. 1A). In both species, the enzyme's prominent cytosolic GT domain protrudes from the micelle-embedded TM segment. Yet, only the trimeric complexes contain an additional stalk extending from the GT domains, which is formed by PttCesA8's NTD (see below) (Fig. 1, A and B).
We focused our structural analysis on the trimeric PttCesA8 particles. Classification in 2 and 3-dimensions, $a b$ initio model building, and non-uniform and local refinement in cryoSPARC (18) with applied 3-fold symmetry generated an excellent density map allowing model building of the trimer excluding its N-terminal stalk (residues 1-156) (Fig. 1C and fig. S2). The GT domain map significantly exceeds the overall estimated resolution of $3.5 \AA$ as essentially all side chains are resolved (fig. S3A). Within the TM segment, aromatic residues as well as comparisons with the bacterial BcsA structure (fig. S4) (19) guided model building. Unstructured regions include residues 548-609 of the CSR domain, 847-868 as part of the Gating Loop, and 959-978 at the extreme C terminus.

PttCesA8's large cytosolic domain is flanked $\mathrm{N}$ - and C-terminally by two and five TM helices, respectively (Fig. 1D). TM helices 1-6 cluster around three cytosolic interface helices (IF1-3) similar to BcsA from R. sphaeroides (19) (Fig. 1D and fig. S4B). TM topology prediction algorithms frequently predict IF3 of the bacterial and plant enzymes as a proper TM helix, thereby giving rise to 8 predicted TM helices in plant CesAs with inverted topologies of TM helices 5-7 (fig. S4A). Yet, IF3 sits on top of IF1 and IF2 on the cytosolic membrane side and rests against TM helices 3,5 and 6 within the bilayer, which stabilizes the helix at the cytosolic water-lipid interface (fig. S4, A and B), as observed in the bacterial enzyme (19). The C-terminal TM helix 7 (residues 938-958) packs into a groove formed by TM helices 4 and 6 as well as IF3 of a neighboring subunit, thereby completing the TM architecture (Fig. 1C). The extracellular C-terminal residues $959-978$ are poorly ordered and not included in the model. Most CesAs' contain a Cys residue at or close to their C-termini (Cys978 in PttCesA8), which could be involved in disulfide bond formation or post-translationally modified, as recently suggested (20). The channel generated by PttCesA8's TM helices accommodates a nascent cellulose chain at least 5 sugar units long (Fig. 1D and fig. S3B). Its non-reducing terminal sugar stacks against Trp718 of the conserved 'QxxRW' motif near the active site (see below and fig. S4, C and D).

\section{The intracellular catalytic and plant specific domains}

PttCesA8's intracellular catalytic domain adopts a classical GT-A fold consisting of a 7 -stranded $\beta$-sheet surrounded by six $\alpha$-helices that create an active site cleft for substrate and acceptor binding (Fig. 1D and fig. S4C). The plant-specific regions, however, protrude from this core in agreement with earlier modeling studies (21). While the PCR belongs to the region of highest map quality, the CSR is partially disordered, with a central region of 62 residues not included in the model.

The PCR (residues 301-418) is inserted into the GT-A fold after D294 of the conserved substrate-binding 'DDG' motif 
$(19,22)$ (Fig. 1D and fig. S5). It contains two anti-parallel $\alpha$ helices connected by a $\sim 20$ residue long linker. PttCesA8's PCR is structurally identical to the corresponding region of rice CesA8, previously crystallized as an isolated domain (23), with which it shares $81 \%$ sequence identity. Past the last $\alpha$ helix, the PCR is connected to the GT-A core via a loop and a short $\beta$-strand that extends GT-A's central $\beta$-sheet (Fig. 1D).

The CSR (residues 542 to 665 ) is inserted into a peripheral loop of the GT-A core forming helical and disordered regions (fig. S5). The helical sub-domain, residues 618-665, contains three short helices, of which the C-terminal helix (residues 640-653) packs into a groove formed by the C-terminal $\beta$ strand of the PCR and $\alpha$-helix 2 of the GT-A core (Fig. 1D). The CSR harbors four cysteine residues within its disordered region close to its N-terminal connection with the GT-A core. Acylation of these residues has recently been implicated in CesA trafficking to the plasma membrane (20). The last resolved residue at the beginning of the CSR, Ser547, is only $\sim 20 \AA$ away from the putative cytosolic water-lipid interface, consistent with membrane-association of the acylated CSR under physiological conditions.

\section{The PCR is a trimerization domain}

Within the trimer, the PttCesA8 protomers are related by a 3fold symmetry axis that runs perpendicular to the plane of the membrane. The complex extends about $90 \AA$ along the membrane normal and has a broad intracellular layer approximately $120 \AA$ in diameter, followed by a narrower TM segment about $75 \AA$ wide (Fig. 1C). Excluding the N-terminal stalk, intermolecular contacts between the subunits are limited to interactions of the C-terminal TM-7 with a neighboring subunit as well as the trimerization of the cytosolic PCRs (Fig. 2A).

PttCesA8 trimerization critically depends on the PCR. In particular, specific intermolecular interactions of the PCR's second $\alpha$-helix (PCR-H2) together with the preceding loop stabilize the trimer interface (Fig. 2A and fig. S5). First, the loop region of subunit ' $A$ ' (residues 339-348) interacts with a hydrophobic C-terminal segment of PCR-H2 in subunit 'B' (residues 359-363). Second, the loop of subunit B contacts the PCR-H2 hydrophobic segment in subunit ' $\mathrm{C}$ ', and, third, the loop of subunit $\mathrm{C}$ interacts with the hydrophobic PCR-H2 segment of subunit A, thereby forming a triangular PCR interface (Fig. 2B). A similar trimeric PCR arrangement is observed in crystals of the isolated rice CesA8 PCR, in which 3 PCR copies are related by crystallographic symmetry (23) (fig. S6A).

While the PCR interactions are primarily based on hydrophobic contacts and shape complementarity, the domains create a significant electropositive trimer interface (Fig. 2C). From each PCR-H2, Arg352 and Arg356 point directly toward the 3-fold symmetry axis and Arg351 and Lys355 are similarly positioned on the opposite side of PCR-H2. Ser333, right above the Lys355/Arg356 pair, has been predicted to be phosphorylated in Arabidopsis thaliana (At) CesA4, which could complement the electropositive environment (Fig. 2C) (24). We observe unidentified cone-shaped density approximately $10 \AA$ long and $6 \AA$ wide on the membrane-proximal and small spherical density on the membrane-distal side of the PCR trimer (Fig. 2B). The apparent pseudo 3-fold symmetric shape of the density is maintained even after refining the data without imposed symmetry, suggesting that it could be a small molecule bound in three different orientations (discussed below).

\section{The CesA8 trimer forms three cellulose channels}

TM-helices 1-6, IF3, and TM-7 of a neighboring subunit form a continuous channel across the membrane that accommodates the nascent cellulose chain (Fig. 3, A and B). The polymer is best resolved extending from the active site, yet disconnected density near the extracellular channel exit indicates the presence of a longer, yet flexible, chain (Fig. 3A and fig. S3B). The polymer must have been synthesized in the expression host and co-purified with the enzyme, as also observed in bacterial BcsA (19).

The TM channel begins right above the active site, with Trp718 of IF2, Phe513 of IF1, as well as Val529 and Gln494 forming the portal (Fig. 3C). The channel is lined with aromatic and hydrophilic residues that likely facilitate glucan translocation. All resolved glucosyl units are coordinated by aromatic residues via $\mathrm{CH}-\pi$ stacking interactions as well as contacts to hydrophilic residues through their equatorial hydroxyl groups (Fig. 3C and fig. S5).

The extracellular channel exit is formed by the TM-3/TM4 loop and TM- 6 of one protomer as well as TM-7 from the neighboring subunit (Figs. 1B and 3B). The TM channel is curved toward the trimer's 3 -fold symmetry axis and forms a profound lateral exit within the extracellular membrane leaflet toward the trimer center (Fig. 3B). Therefore, cellulose chains simultaneously synthesized by a PttCesA8 trimer are extracellularly released within approximately $30 \AA$ from each other, which may facilitate their alignment into a trimeric cellulose 'protofibril (Fig. 3D), perhaps represented by the fibrillar reaction product produced by detergent-solubilized PttCesA8 (fig. S1D).

\section{Architecture of the full-length PttCesA8 trimer}

Two-dimensional classification and our low-resolution shape reconstruction of the PttCesA8 trimer revealed the presence of a stalk protruding from the cytosolic domains (Fig. 1, A and B). The stalk is about $70 \AA$ long and roughly aligns with the 3 -fold symmetry axis. It represents CesA's NTD, which, in land plants, contains a zinc-coordinating RING-like domain at the extreme $\mathrm{N}$ terminus, followed by a hypervariable 
region 90-150 amino acids long (10), which is significantly shorter in CesA8 enzymes compared to other isoforms.

Local refinement of the stalk region (fig. S2) improved the map resolution to approximately $5 \AA$, revealing a central helical coiled-coil of three $\alpha$-helices together with an extended region at its tip (Fig. 4A). Additional short, likely helical, segments are discernable near the interface with the electropositive PCR trimer where both domains interact. The conserved PCR residues D357 and K349 are suitably positioned to mediate these interactions (Fig. 4A).

The function of the NTD is currently unknown, yet dimerization and zinc ion coordination of the isolated $\mathrm{N}$ terminus from cotton CesAl has been suggested (15). PttCesA8's isolated NTD (residues 1-160) also purifies in a monomer-dimer equilibrium, but we can also detect a small fraction of a trimeric species by gel filtration chromatography and Westernblotting, along with additional, higher molecular weight species (fig. S6, B and C). These results suggest a propensity of the NTD to self-associate, which is consistent with the trimeric form observed in the EM structure.

The NTD is connected to the well-ordered GT-A core via residues 157-165 that form a two-stranded anti-parallel $\beta$ sheet with the TM-2 - GT-A connection (Fig. 1D). Considering that the NTD's RING-like domain is formed by the most Nterminal $\sim 50$ residues and is followed by a helical region about 30 residues long, the unresolved linker would be $\sim 70$ to 90 residues long (depending on the isoform) (Fig. 4A).

Cellulose Synthase Interactive (CSI) proteins have been shown to interact with CesAs involved in primary cell wall formation as well as microtubules, thereby likely facilitating the directional deposition of cellulose fibrils on the cell surface (25-27). We observe binding of full-length PttCesA8 as well as its soluble NTD to CSI-1-coated beads (fig. S6D), suggesting the N-terminal stalk could mediate protein-protein interactions implicated in CSC microtubule tethering.

\section{Implications of CesA mutations and phosphorylation}

Several CesA mutations have been reported to either alter the overall amount of cellulose produced, its crystallinity, i.e., CMF organization, or to confer resistances to herbicides. Localizing these substitutions in the PttCesA8 trimer structure provides insights into their mode of action. For example, mutations conferring resistance to the herbicides quinoxyphen and isoxaben in AtCesA1, 3 and 6 (CesA1:A903V for quinoxyphen and CesA3:G998D and CesA6:R1064W for isoxaben resistance) map to PttCesA8's I800, G909, and K956, respectively, of which G909 and K956 frame the extracellular channel exit $(28,29)$ (fig. S7A). I800 is at the center of TM-4 and packs against TM-7 from the neighboring subunit. An additional isoxaben resistance mutation, T942I in AtCesA3, localizes to the conserved FxVTxK motif of the gating loop, a region that is disordered in our substrate-free structure, similar to observations with bacterial BcsA $(28,30)$ (figs. S4A and S7A).

Further, several phosphorylation sites have been identified in AtCesAs, potentially affecting CesA activity and degradation $(24,31)$. Interestingly, with the exception of S160 near the connection of the N-terminal linker with TM-1 (Fig. 4A) and S333 at the PCR trimer interface discussed above (Fig. 2B), most sites localize to poorly ordered NTD and CSR regions, where they could affect interactions with other binding partners (fig. S7B).

\section{Discussion}

Most plants express several different CesA isoforms that range in sequence identity from $\sim 55$ to $>68 \%$ and are not functionally equivalent. Among the secondary cell wall CesAs (CesA4, 7 and 8), CesA8 is more highly expressed in aspen tension wood compared to isoforms 4 and 7 , suggesting that CesA8 may play a particular role in secondary cell wall formation (32). Yet the isoforms interact, perhaps by forming heterotrimeric complexes similar to the PttCesA8 homotrimer (33). PttCesA8 differs from PttCesA4 and 7 mostly in a shortened hypervariable region of the NTD that forms part of the stalk as well as within the CSR (Fig. 4A and fig. S8). Mapping all conserved residues between the three isoforms onto our PttCesA8 trimer structure reveals that the complex interfaces formed by the PCR and TM region are nearly identical (fig. S8). This may explain why recombinantly expressed PttCesA8 assembles into homo-trimers, yet in vivo, co-expression of different CesA isoforms may favor hetero-oligomer formation.

Our cryo-EM structure suggests that CesA catalytic activity requires its assembly into at least trimeric species. The TM regions of CesA subunits may assemble into trimers upon insertion in the ER membrane, and the tight interaction of the intracellular PCR domains could require posttranslational modifications or binding of a ligand. The electropositive character of the PCR trimer interface (Fig. 2C) almost certainly requires a stabilizing counter charge, which, in turn, could control PCR assembly and, potentially, activity of the trimeric complex. Indeed, the trimeric structure of the isolated rice PCR domain is stabilized by a phosphate ion (fig. S6A) (23). We also observe additional density at this interface significantly larger than a phosphate ion that readily accommodates a dinucleotide, which could provide the necessary counter charge (Fig. 2B and fig. S9).

Cellulose microfibrils have been observed to align with cortical microtubules and tracks of CSCs during cellulose biosynthesis also align with this network $(34,35)$. These connections are established by CSI proteins that tether CesAs to the microtubule network (34). We reveal that PttCesA8's NTD binds CSI-1 in vitro and its protrusion into the cytosol would be ideal to also mediate these interactions in vivo. Because 
the NTD's RING-like domain is shared by all CesA isoforms, an interaction of CSI-1 with this domain seems most likely. However, because CSI proteins have been shown to interact with primary cell wall CesAs in yeast two-hybrid screens using the GT domain (including CSR and PCR) as bait, we cannot exclude multiple interactions with the NTD and GT domain.

The membrane-embedded region of the PttCesA8 trimer superimposes neatly with the rosette's repeat unit structure (16) (fig. S10A). Therefore, our data support earlier proposals of rosettes consisting of hexamers of CesA trimers $(16,36)$. We propose that trimeric CesA complexes produce cellulose protofibrils containing three glucan chains that, in the context of a rosette, assemble into microfibrils containing 18 cellulose chains (Fig. 4B and fig. S10). Recent modeling studies support this hypothesis (37).

\section{REFERENCES AND NOTES}

1. D. J. Nevins, Sugars: Their origin in photosynthesis and subsequent biological interconversions. Am. J. Clin. Nutr. 61, 915S-921S (1995). doi:10.1093/ajcn/61.4.915S Medline

2. K. Keegstra, Plant cell walls. Plant Physiol. 154, 483-486 (2010). doi:10.1104/pp.110.161240 Medline

3. J. C. Duchesne, D. W. Larson, Cellulose and the evolution of plant life. BioScience 39, 238-241 (1989). doi:10.2307/1311160

4. L. L. Lairson, B. Henrissat, G. J. Davies, S. G. Withers, Glycosyltransferases: Structures, functions, and mechanisms. Annu. Rev. Biochem. 77, 521-555 (2008). doi:10.1146/annurev.biochem.76.061005.092322 Medline

5. B. L. Cantarel, P. M. Coutinho, C. Rancurel, T. Bernard, V. Lombard, B. Henrissat, The Carbohydrate-Active EnZymes database (CAZy): An expert resource for glycogenomics. Nucleic Acids Res. 37 (Database), D233-D238 (2009). doi:10.1093/nar/gkn663 Medline

6. Y. Bi, C. Hubbard, P. Purushotham, J. Zimmer, Insights into the structure and function of membrane-integrated processive glycosyltransferases. Curr. Opin. Struct. Biol. 34, 78-86 (2015). doi:10.1016/i.sbi.2015.07.008 Medline

7. J. L. Morgan, J. T. McNamara, M. Fischer, J. Rich, H.-M. Chen, S. G. Withers, J. Zimmer, Observing cellulose biosynthesis and membrane translocation in crystallo. Nature 531, 329-334 (2016). doi:10.1038/nature16966 Medline

8. M. Iguchi, S. Yamanaka, A. Budhiono, Bacterial cellulose; a masterpiece of nature's arts. J. Mater. Sci. 35, 261-270 (2000). doi:10.1023/A:1004775229149

9. O. A. McCrate, X. Zhou, C. Reichhardt, L. Cegelski, Sum of the parts: Composition and architecture of the bacterial extracellular matrix. J. Mol. Biol. 425, 4286-4294 (2013). doi:10.1016/i.jmb.2013.06.022 Medline

10. H. E. McFarlane, A. Döring, S. Persson, The cell biology of cellulose synthesis. Annu. Rev. Plant Biol. 65, 69-94 (2014). doi:10.1146/annurev-arplant-050213$\underline{040240}$ Medline

11. S. H. Cho, P. Purushotham, C. Fang, C. Maranas, S. M. Díaz-Moreno, V. Bulone, J. Zimmer, M. Kumar, B. T. Nixon, Synthesis and self-assembly of cellulose microfibrils from reconstituted cellulose synthase. Plant Physiol. 175, 146-156 (2017). doi:10.1104/pp.17.00619 Medline

12. P. Purushotham, S. H. Cho, S. M. Díaz-Moreno, M. Kumar, B. T. Nixon, V. Bulone, J. Zimmer, A single heterologously expressed plant cellulose synthase isoform is sufficient for cellulose microfibril formation in vitro. Proc. Natl. Acad. Sci. U.S.A. 113, 11360-11365 (2016). doi:10.1073/pnas.1606210113 Medline

13. C. Somerville, Cellulose synthesis in higher plants. Annu. Rev. Cell Dev. Biol. 22, 53-78 (2006). doi:10.1146/annurev.cellbio.22.022206.160206 Medline

14. C. E. Vergara, N. C. Carpita, $\beta$-D-glycan synthases and the CesA gene family: Lessons to be learned from the mixed-linkage $(1 \rightarrow 3),(1 \rightarrow 4) \beta$-D-glucan synthase. Plant Mol. Biol. 47, 145-160 (2001). doi:10.1023/A:1010631431620 Medline

15. I. Kurek, Y. Kawagoe, D. Jacob-Wilk, M. Doblin, D. Delmer, Dimerization of cotton fiber cellulose synthase catalytic subunits occurs via oxidation of the zinc-binding domains. Proc. Natl. Acad. Sci. U.S.A. 99, 11109-11114 (2002). doi:10.1073/pnas.162077099 Medline

16. B. T. Nixon, K. Mansouri, A. Singh, J. Du, J. K. Davis, J.-G. Lee, E. Slabaugh, V. G. Vandavasi, H. O'Neill, E. M. Roberts, A. W. Roberts, Y. G. Yingling, C. H. Haigler, Comparative structural and computational analysis supports eighteen cellulose synthases in the plant cellulose synthesis complex. Sci. Rep. 6, 28696 (2016). doi:10.1038/srep28696 Medline

17. M. C. Jarvis, Cellulose biosynthesis: Counting the chains. Plant Physiol. 163, 14851486 (2013). doi:10.1104/pp.113.231092 Medline

18. A. Punjani, J. L. Rubinstein, D. J. Fleet, M. A. Brubaker, cryoSPARC: Algorithms for rapid unsupervised cryo-EM structure determination. Nat. Methods 14, 290-296 (2017). doi:10.1038/nmeth.4169 Medline

19. J. L. Morgan, J. Strumillo, J. Zimmer, Crystallographic snapshot of cellulose synthesis and membrane translocation. Nature 493, 181-186 (2013). doi:10.1038/nature11744 Medline

20. M. Kumar, R. Wightman, I. Atanassov, A. Gupta, C. H. Hurst, P. A. Hemsley, S. Turner, S-Acylation of the cellulose synthase complex is essential for its plasma membrane localization. Science 353, 166-169 (2016). doi:10.1126/science.aaf4009 Medline

21. L. Sethaphong, C. H. Haigler, J. D. Kubicki, J. Zimmer, D. Bonetta, S. DeBolt, Y. G. Yingling, Tertiary model of a plant cellulose synthase. Proc. Natl. Acad. Sci. U.S.A. 110, 7512-7517 (2013). doi:10.1073/pnas.1301027110 Medline

22. I. M. Saxena, R. M. Brown Jr., T. Dandekar, Structure-function characterization of cellulose synthase: Relationship to other glycosyltransferases. Phytochemistry 57, 1135-1148 (2001). doi:10.1016/S0031-9422(01)00048-6 Medline

23. P. S. Rushton, A. T. Olek, L. Makowski, J. Badger, C. N. Steussy, N. C. Carpita, C. V. Stauffacher, Rice cellulose synthaseA8 plant-conserved region is a coiled-coil at the catalytic core entrance. Plant Physiol. 173, 482-494 (2017). doi:10.1104/pp.16.00739 Medline

24. T. L. Speicher, P. Z. Li, I. S. Wallace, Phosphoregulation of the plant cellulose synthase complex and cellulose synthase-like proteins. Plants 7, 52 (2018). doi:10.3390/plants7030052 Medline

25. S. Li, L. Lei, C. R. Somerville, Y. Gu, Cellulose synthase interactive protein 1 (CSI1) links microtubules and cellulose synthase complexes. Proc. Natl. Acad. Sci. U.S.A. 109, 185-190 (2012). doi:10.1073/pnas.1118560109 Medline

26. L. Lei, S. Li, J. Du, L. Bashline, Y. Gu, Cellulose synthase INTERACTIVE3 regulates cellulose biosynthesis in both a microtubule-dependent and microtubuleindependent manner in Arabidopsis. Plant Cell 25, $4912-4923$ (2013). doi:10.1105/tpc. 113.116715 Medline

27. Y. Gu, N. Kaplinsky, M. Bringmann, A. Cobb, A. Carroll, A. Sampathkumar, T. I. Baskin, S. Persson, C. R. Somerville, Identification of a cellulose synthaseassociated protein required for cellulose biosynthesis. Proc. Natl. Acad. Sci. U.S.A. 107, 12866-12871 (2010). doi:10.1073/pnas.1007092107 Medline

28. W. R. Scheible, R. Eshed, T. Richmond, D. Delmer, C. Somerville, Modifications of cellulose synthase confer resistance to isoxaben and thiazolidinone herbicides in Arabidopsis Ixr1 mutants. Proc. Natl. Acad. Sci. U.S.A. 98, 10079-10084 (2001). doi:10.1073/pnas.191361598 Medline

29. D. M. Harris, K. Corbin, T. Wang, R. Gutierrez, A. L. Bertolo, C. Petti, D.-M. Smilgies, J. M. Estevez, D. Bonetta, B. R. Urbanowicz, D. W. Ehrhardt, C. R. Somerville, J. K. C. Rose, M. Hong, S. Debolt, Cellulose microfibril crystallinity is reduced by mutating C-terminal transmembrane region residues CESA1A903V and CESA3T942I of cellulose synthase. Proc. Natl. Acad. Sci. U.S.A. 109, 4098-4103 (2012). doi:10.1073/pnas.1200352109 Medline

30. J. L. W. Morgan, J. T. McNamara, J. Zimmer, Mechanism of activation of bacterial cellulose synthase by cyclic di-GMP. Nat. Struct. Mol. Biol. 21, 489-496 (2014). doi:10.1038/nsmb.2803 Medline

31. S. Chen, H. Jia, H. Zhao, D. Liu, Y. Liu, B. Liu, S. Bauer, C. R. Somerville, Anisotropic cell expansion is affected through the bidirectional mobility of cellulose synthase complexes and phosphorylation at two critical residues on CESA3. Plant Physiol. 171, 242-250 (2016). doi:10.1104/pp.15.01874 Medline

32. X. Zhang, P. G. Dominguez, M. Kumar, J. Bygdell, S. Miroshnichenko, B. Sundberg, G. Wingsle, T. Niittylä, Cellulose synthase stoichiometry in aspen differs from Arabidopsis and Norway spruce. Plant Physiol. 177, 1096-1107 (2018). doi:10.1104/pp.18.00394 Medline

33. J. Timmers, S. Vernhettes, T. Desprez, J.-P. Vincken, R. G. F. Visser, L. M. Trindade, Interactions between membrane-bound cellulose synthases involved in the 
synthesis of the secondary cell wall. FEBS Lett. 583, 978-982 (2009). doi:10.1016/i,febslet.2009.02.035 Medline

34. M. Bringmann, B. Landrein, C. Schudoma, O. Hamant, M.-T. Hauser, S. Persson, Cracking the elusive alignment hypothesis: The microtubule-cellulose synthase nexus unraveled. Trends Plant Sci. 17, 666-674 (2012). doi:10.1016/i.tplants.2012.06.003 Medline

35. M. Woodley, A. Mulvihill, M. Fujita, G. O. Wasteneys, Exploring microtubuledependent cellulose-synthase-complex movement with high precision particle tracking. Plants 7, 53 (2018). doi:10.3390/plants7030053 Medline

36. V. G. Vandavasi, D. K. Putnam, Q. Zhang, L. Petridis, W. T. Heller, B. T. Nixon, C. H. Haigler, U. Kalluri, L. Coates, P. Langan, J. C. Smith, J. Meiler, H. O'Neill, A structural study of CESA1 catalytic domain of Arabidopsis cellulose synthesis complex: Evidence for CESA trimers. Plant Physiol. 170, 123-135 (2016). doi:10.1104/pp.15.01356 Medline

37. J. D. Kubicki, H. Yang, D. Sawada, H. O'Neill, D. Oehme, D. Cosgrove, The shape of native plant cellulose microfibrils. Sci. Rep. 8, 13983 (2018). doi:10.1038/s41598018-32211-w Medline

38. B. K. Ho, F. Gruswitz, HOLLOW: Generating accurate representations of channel and interior surfaces in molecular structures. BMC Struct. Biol. 8, 49 (2008). doi:10.1186/1472-6807-8-49 Medline

39. M. Kumar, S. Thammannagowda, V. Bulone, V. Chiang, K.-H. Han, C. P. Joshi, S. D. Mansfield, E. Mellerowicz, B. Sundberg, T. Teeri, B. E. Ellis, An update on the nomenclature for the cellulose synthase genes in Populus. Trends Plant Sci. 14, 248-254 (2009). doi:10.1016/i.tplants.2009.02.004 Medline

40. E. F. Pettersen, T. D. Goddard, C. C. Huang, G. S. Couch, D. M. Greenblatt, E. C. Meng, T. E. Ferrin, UCSF Chimera-A visualization system for exploratory research and analysis. J. Comput. Chem. 25, 1605-1612 (2004). doi:10.1002/jcc.20084 Medline

41. P. Emsley, K. Cowtan, Coot: Model-building tools for molecular graphics. Acta Crystallogr. D Biol. Crystallogr. 60, 2126-2132 (2004). doi:10.1107/S0907444904019158 Medline

42. P. D. Adams, P. V. Afonine, G. Bunkóczi, V. B. Chen, I. W. Davis, N. Echols, J. J. Headd, L.-W. Hung, G. J. Kapral, R. W. Grosse-Kunstleve, A. J. McCoy, N. W. Moriarty, R. Oeffner, R. J. Read, D. C. Richardson, J. S. Richardson, T. C. Terwilliger, P. H. Zwart, PHENIX: A comprehensive Python-based system for macromolecular structure solution. Acta Crystallogr. D Biol. Crystallogr. 66, 213221 (2010). doi:10.1107/S0907444909052925 Medline

43. PyMol, in DeLano Scientific (San Carlos, CA, 2002).

44. B. K. Ho, F. Gruswitz, HOLLOW: Generating accurate representations of channel and interior surfaces in molecular structures. BMC Struct. Biol. 8, 49 (2008). doi:10.1186/1472-6807-8-49 Medline

45. A. Caño-Delgado, S. Penfield, C. Smith, M. Catley, M. Bevan, Reduced cellulose synthesis invokes lignification and defense responses in Arabidopsis thaliana. Plant J. 34, 351-362 (2003). doi:10.1046/i.1365-313X.2003.01729.x Medline

46. Z. Chen, X. Hong, H. Zhang, Y. Wang, X. Li, J.-K. Zhu, Z. Gong, Disruption of the cellulose synthase gene, AtCesA8/IRX1, enhances drought and osmotic stress tolerance in Arabidopsis. Plant J. 43, 273-283 (2005). doi:10.1111/i.1365313X.2005.02452.x Medline

47. M. Fujita, R. Himmelspach, J. Ward, A. Whittington, N. Hasenbein, C. Liu, T. T. Truong, M. E. Galway, S. D. Mansfield, C. H. Hocart, G. O. Wasteneys, The anisotropy1 D604N mutation in the Arabidopsis cellulose synthasel catalytic domain reduces cell wall crystallinity and the velocity of cellulose synthase complexes. Plant Physiol. 162, 74-85 (2013). doi:10.1104/pp.112.211565 Medline

48. S. Rubio-Díaz, J. M. Pérez-Pérez, R. González-Bayón, R. Muñoz-Viana, N. Borrega, G. Mouille, D. Hernández-Romero, P. Robles, H. Höfte, M. R. Ponce, J. L. Micol, Cell expansion-mediated organ growth is affected by mutations in three EXIGUA genes. PLOS ONE 7, e36500 (2012). doi:10.1371/journal.pone.0036500 Medline

49. B. Zhang, L. Deng, Q. Qian, G. Xiong, D. Zeng, R. Li, L. Guo, J. Li, Y. Zhou, A missense mutation in the transmembrane domain of CESA4 affects protein abundance in the plasma membrane and results in abnormal cell wall biosynthesis in rice. Plant Mol. Biol. 71, 509-524 (2009). doi:10.1007/s11103-009-9536-4 Medline

\section{ACKNOWLEDGMENTS}

We thank Sung Hyun Cho, Tracy Nixon, and Carol Bator for support using the Huck Institute cryo-EM facility at the Pennsylvania State University. We thank
Georgios Skiniotis for discussions and are grateful for support from Kelly Dryden and Michael Purdy from the MEMC at the University of Virginia. We are grateful to Daniel Cosgrove and Balasubramanyam Chittoor for comments on the manuscript. Funding: This work was supported as part of The Center for Lignocellulose Structure and Formation, an Energy Frontier Research Center funded by the US Department of Energy, Office of Science, Basic Energy Sciences (Award Number DESC0001090). MEMC is funded by NIH Recovery grant 1G20RR31199. The MEMC Titan Krios and Gatan K3/GIF detector were funded through NIH grants SIG S10-RR025067 and U24-GM116790, respectively. Author contributions: P.P. established all expression, purification, and activity assay protocols and prepared materials for cryo grid preparation. R.H. prepared and screened cryo grids and collected all data sets. J.Z. processed the data and built and refined the model. All authors contributed to preparing the manuscript. Competing interests: The authors declare no competing interests.

Data and materials availability: EM maps have been deposited at the EMDB (EMD-21820) and model coordinates have been deposited at the PDB under accessing code 6WLB. All other data are provided in the main text or supplementary materials.

\section{SUPPLEMENTARY MATERIALS}

science.sciencemag.org/cgi/content/full/science.abb2978/DC1

Materials and Methods

Figs. S1 to S10

Table S1

References (39-49)

MDAR Reproducibility Checklist

13 February 2020; accepted 26 June 2020

Published online 9 July 2020

10.1126/science.abb2978 


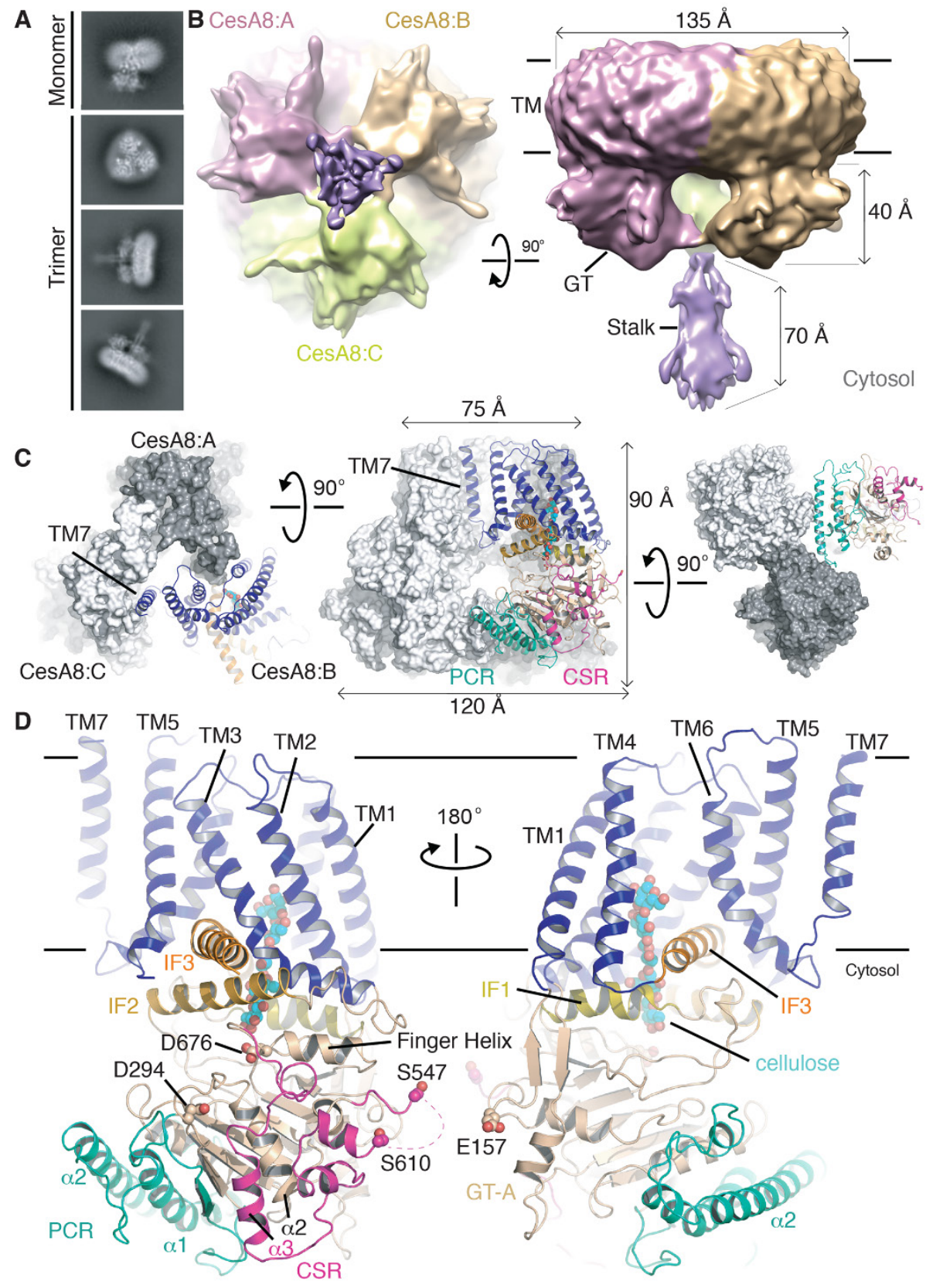

Fig. 1. Structure of PttCesA8. (A) Representative 2D class averages of monomeric and trimeric PttCesA8 particles. (B) Low resolution reconstruction of a full length PttCesA8 trimer shown at a map contour level of 1.86. (C) Trimeric assembly of the PttCesA8 core. Two copies are shown as surfaces colored light and dark gray, respectively. One subunit is shown as a cartoon colored blue, wheat, bluegreen, and magenta for the TM, GT, PCR and CSR domains, respectively. Amphipathic interface helices are colored in shades of orange. (D) Detailed organization of a PttCesA8 monomer. Residues 548 to 609 of the CSR are disordered. Cellulose is shown as cyan and red spheres. 


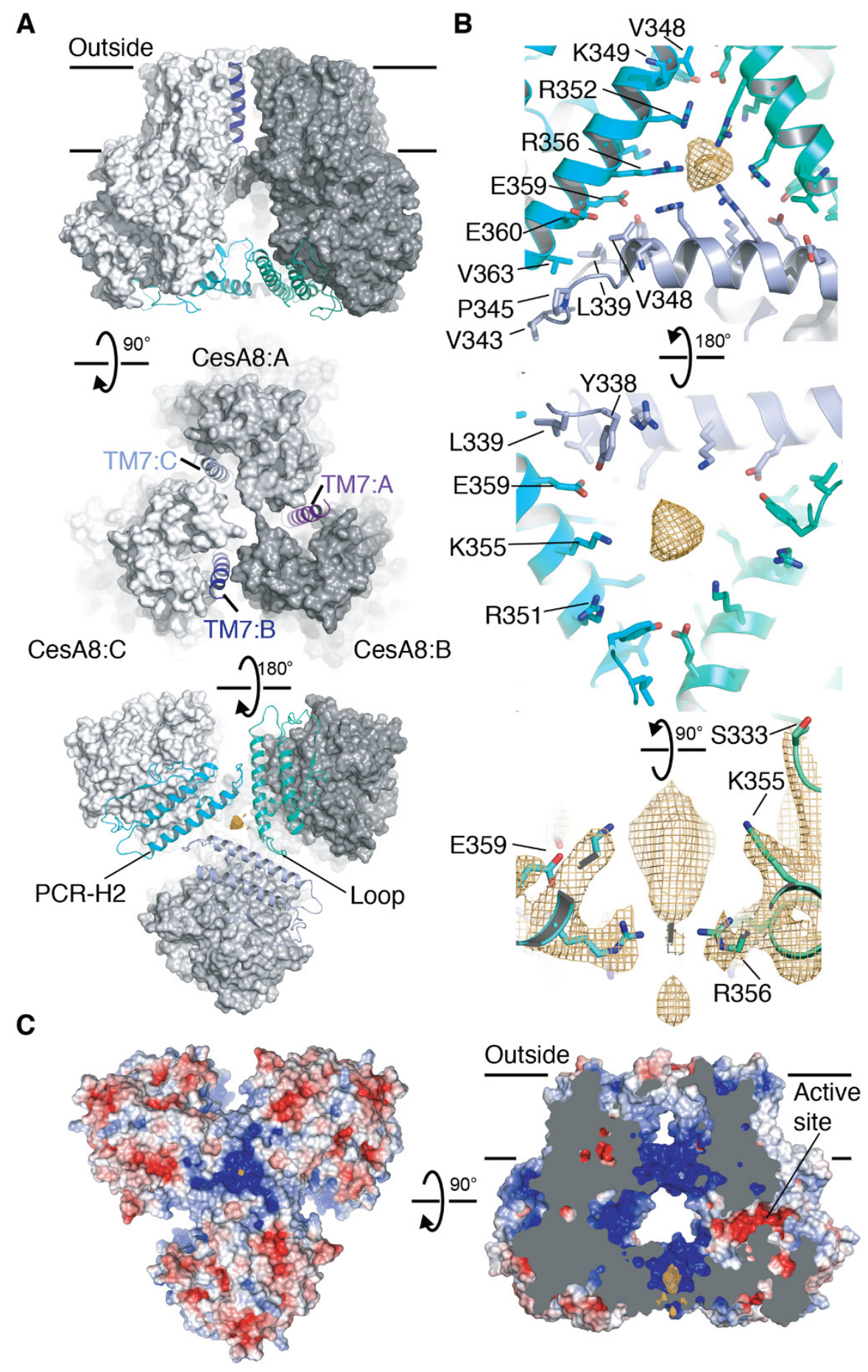

Fig. 2. Trimerization of PttCesA8. (A) Surface representation of the CesA8 trimer with all C-terminal TM helices (TM7) and PCR domains shown as cartoons. (B) Detailed interactions of the PCR trimer interface. Interface residues are shown as sticks and labeled. Unidentified density is shown as an orange mesh (B-factor sharpened (-93) and contoured at $8 \sigma$ ). A larger section of the density map is shown in the bottom panel. (C) Surface electrostatic potential of the PttCesA8 trimer calculated with the APBS plugin in Pymol and colored red to blue ( -5 to $+5 \mathrm{kT}$, respectively). The unidentified density is shown as in (B). 


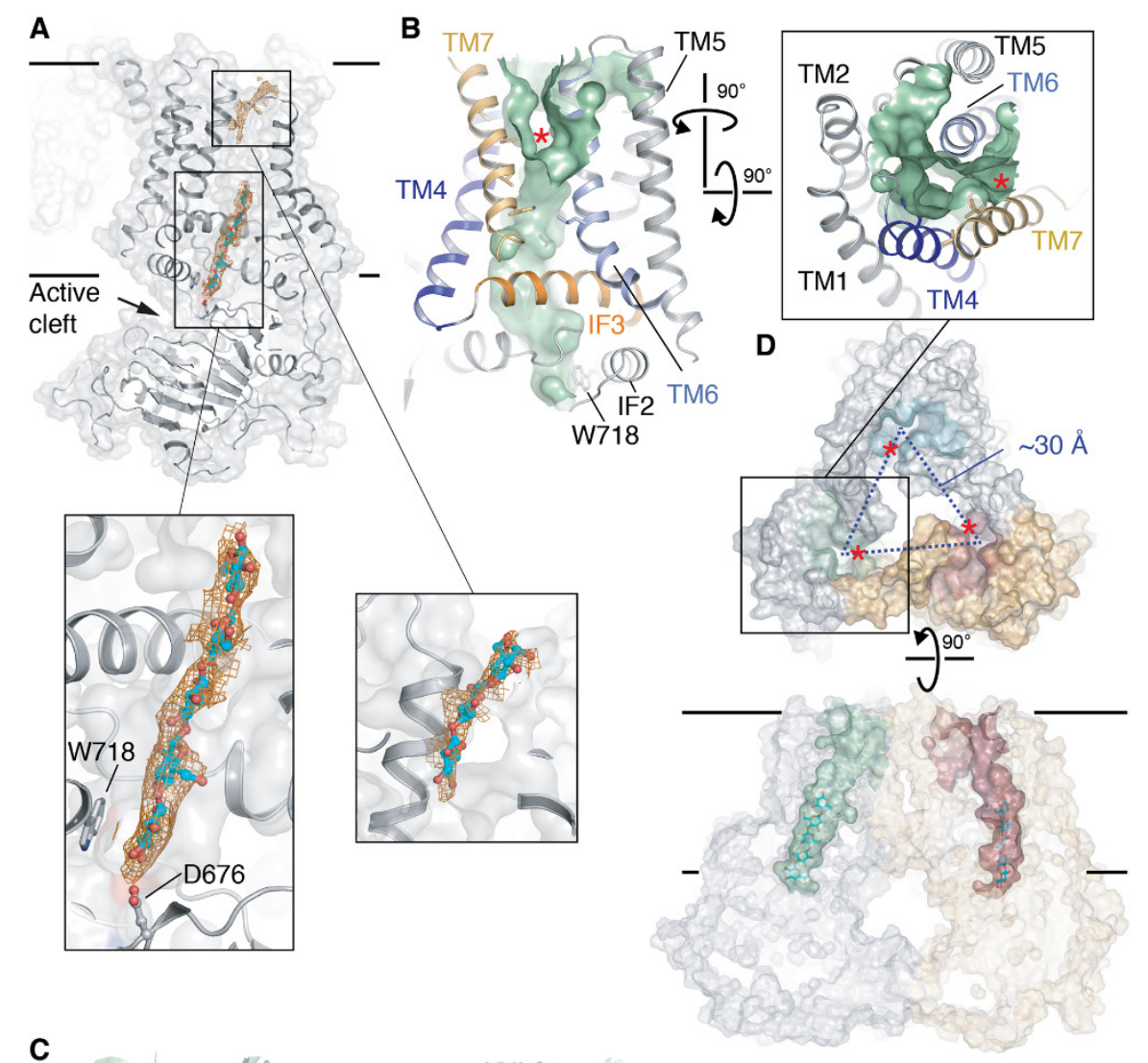

C

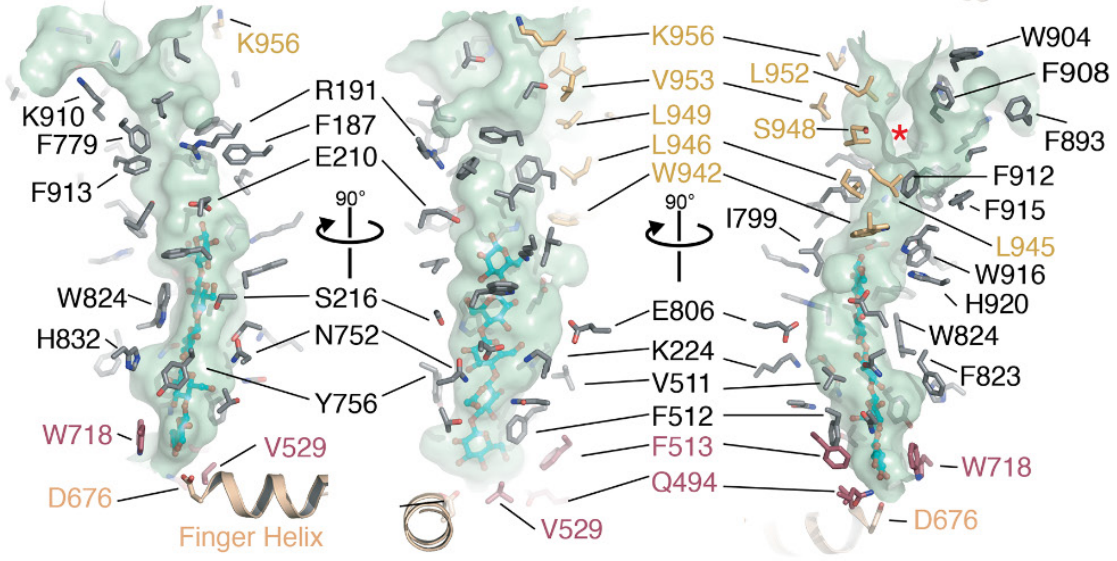

Fig. 3. The PttCesA8 channel contains a nascent cellulose polymer. (A) Each CesA8 subunit contains a glucan chain at least 5 glucose units long. A PttCesA8 subunit is shown as a semitransparent surface and cartoon. Density representing cellulose is shown as a brown mesh. The density near the extracellular channel exit is significantly weaker (shown at $4.5 \sigma$ contour level) compared to the one near the active site (contoured at 5.5 $\sigma$ ) and may represent three glucosyl units (shown as cyan and red ball-and-sticks). (B) PttCesA8 forms a continuous transmembrane channel. Channel dimensions were calculated in Hollow (38) with a probe radius of $1.8 \AA$ after removing cellulose. (C) Cellulose coordination by channel lining residues from two PttCesA8 subunits. Residues contributed by TM7 of another subunit and those lining the cytosolic channel entrance are labeled in wheat and magenta, respectively. Red asterisk: lateral channel exit. (D) Path of the TM channels in the PttCesA8 trimer. One subunit is colored pale orange, the others are shown in gray. 


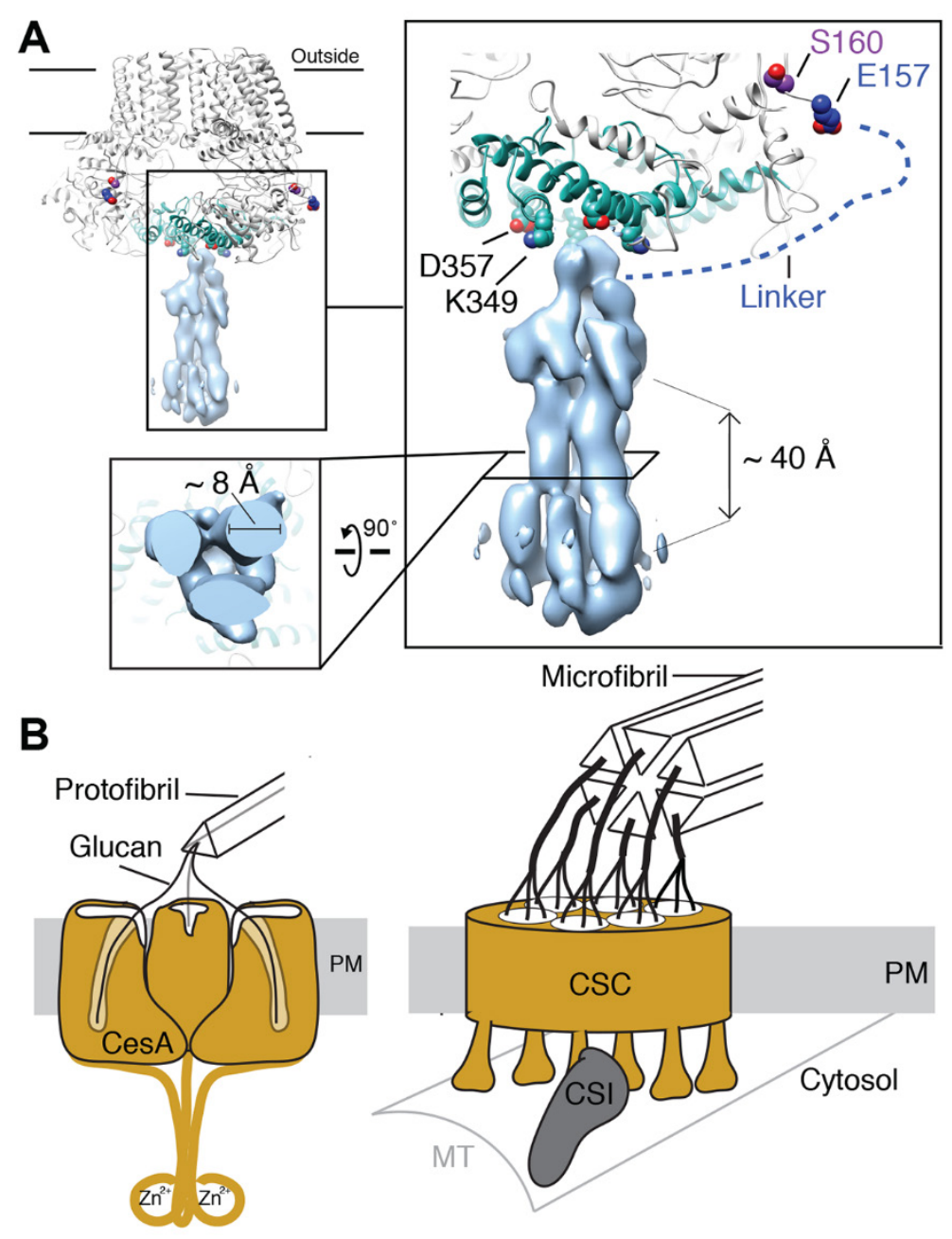

Fig. 4. Structural organization of PttCesA8's stalk and proposed rosette assembly. (A) The CesA8 core structure aligned with the locally refine stalk density [blue surface, contoured at $1.2 \sigma$ after Bfactor sharpening (-200)]. The central stalk region contains three helical segments. (B) Model of cellulose microfibril formation in which trimer CesA assemblies produces protofibrils that align into a CMF in the context of a rosette. Microtubule and CesA interacting proteins may facilitate proper localization and movement of the CSCs in the plasma membrane (PM). 